\title{
Magnetic moments of heavy baryons
}

\author{
M. C. Bañuls, I. Scimemi, J. Bernabéu, V. Giménez, and A. Pich \\ Departamento de Física Teórica, IFIC, Universidad de Valencia-CSIC, E-46100 Burjassot (Valencia), Spain
}

(Received 27 May 1999; published 18 February 2000)

\begin{abstract}
The first nontrivial chiral corrections to the magnetic moments of triplet $(T)$ and sextet $\left(S^{(*)}\right)$ heavy baryons are calculated using heavy hadron chiral perturbation theory. Since magnetic moments of the $T$ hadrons vanish in the limit of infinite heavy quark mass $\left(m_{Q} \rightarrow \infty\right)$, these corrections occur at order $\mathcal{O}\left(1 /\left(m_{Q} \Lambda_{\chi}^{2}\right)\right)$ for $T$ baryons, while for $S^{(*)}$ baryons they are of order $\mathcal{O}\left(1 / \Lambda_{\chi}^{2}\right)$. The renormalization of the chiral loops is discussed, and relations among the magnetic moments of different hadrons are provided. Previous results for $T$ baryons are revised.
\end{abstract}

PACS number(s): 12.39.Fe, 12.39.Hg, 14.20.Lq, 14.20.Mr

\section{INTRODUCTION}

Chiral perturbation theory (ChPT) and heavy quark effective theory (HQET) can be combined together to construct an effective Lagrangian which describes soft interactions of hadrons containing a single heavy quark [1-4]. Electromagnetic interactions can be included in the formalism by gauging a $\mathrm{U}(1)_{E M}$ subgroup of the global $\mathrm{SU}(3)_{L} \otimes \mathrm{SU}(3)_{R}$ symmetry group.

In the limit $m_{u}, m_{d}, m_{s} \rightarrow 0$, the QCD Lagrangian for light quarks has a $\mathrm{SU}(3)_{L} \otimes \mathrm{SU}(3)_{R} \otimes \mathrm{U}(1)_{V}$ symmetry, which is spontaneously broken to $\mathrm{SU}(3)_{V} \otimes \mathrm{U}(1)_{V}$. The lightest particles of the hadronic spectrum, the pseudoscalar octet ( $\pi, K, \bar{K}, \eta)$, can be identified with the corresponding Goldstone bosons. Their low-energy interactions can be analyzed making use of ChPT [5], which is an expansion in terms of momenta and meson masses. Goldstone bosons are parametrized as

$$
\pi=\frac{1}{\sqrt{2}}\left(\begin{array}{ccc}
\sqrt{\frac{1}{2}} \pi^{0}+\sqrt{\frac{1}{6}} \eta & \pi^{+} & K^{+} \\
\pi^{-} & -\sqrt{\frac{1}{2}} \pi^{0}+\sqrt{\frac{1}{6}} \eta & K^{0} \\
K^{-} & \bar{K}^{0} & -\sqrt{\frac{2}{3}} \eta
\end{array}\right),
$$

and appear in the Lagrangian via the exponential representation $\Sigma=\exp \left(2 i \pi / f_{\pi}\right) \equiv \xi^{2}$, being $f_{\pi} \sim 93 \mathrm{MeV}$ the pion decay constant. Under chiral transformations,

$$
\Sigma \rightarrow L \Sigma R^{+}, \quad \xi \rightarrow L \xi h^{+}=h \xi R^{+}
$$

where $L(R)$ are global elements of $\mathrm{SU}(3)_{L(R)}$ and $h$ is a local $\mathrm{SU}(3)_{L+R}$ transformation, which depends both on $\pi$ and on the chiral transformation $(L, R)$. To construct the effective theory, one must write the most general Lagrangian consistent with chiral symmetry involving $\Sigma$ and its derivatives. Chiral symmetry is explicitly broken in QCD by the quark mass term. This can be incorporated in the effective Lagrangian through the light mass matrix $\chi$, which gives rise to a quadratic pseudoscalar mass term. The Lagrangian is then organized as an expansion in powers of $\left(p / \Lambda_{\chi}\right)$ and $\left(m_{q} / \Lambda_{\chi}\right)$, where $p$ is the low pseudoscalar momenta, $m_{q}$ denotes the light quark masses, and $\Lambda_{\chi} \approx 1 \mathrm{GeV}$ is the chiral symmetry breaking scale which suppresses higher-order terms in the effective theory.

On the other side, in the opposite limit, $m_{Q} \rightarrow \infty$, which is useful for $c$ and $b$ quarks, different simplifications occur in the dynamics of heavy-light hadrons. Quark interactions do not change the velocity of the heavy quark inside the hadron, because the momentum exchange is of order $\delta P \sim \Lambda_{\mathrm{QCD}}$ $\ll m_{Q}$. In the hadron rest frame, the heavy quark acts as a static color source which interacts with the light degrees of freedom. This interaction is independent of the mass and spin of the heavy quark, and thus the hadron dynamics shows $\mathrm{SU}(2)$ spin symmetry and $\mathrm{SU}\left(N_{f}\right)$ flavor symmetry (for $N_{f}$ heavy flavors). HQET [6] is an effective field theory for QCD which makes this symmetry manifest in the $m_{Q} \rightarrow \infty$ limit and describes the dynamics of hadrons containing a heavy quark at momenta much lower than $m_{Q}$. The effective baryon fields are labeled by their velocities, and their mass is removed from the baryon momentum $P$. Derivatives on the baryon fields produce powers of residual momentum $\left(k / m_{Q}\right) \ll\left(P / m_{Q}\right)$.

In some kinematical regions, which are not far from the chiral and heavy quark limits, both approaches can be simultaneously used. Baryons containing a heavy quark, in the $m_{Q} \rightarrow \infty$ limit, can emit and absorb light pseudoscalar mesons without changing its velocity, $v$. In heavy hadron chiral perturbation theory (HHCPT), one constructs an effective Lagrangian whose basic fields are heavy hadrons and light mesons. In Ref. [7], the formalism is extended to include also electromagnetism. We use this hybrid effective Lagrangian to calculate the magnetic moments (MMs) of some baryons containing a $c$ or a $b$ quark.

In Sec. II we review the needed HHCPT formalism, introduced in Ref. [7]: the effective fields representing $S$ and $T$ baryons, the lowest-order chiral Lagrangian and its $\mathcal{O}\left(1 / m_{Q}\right), \mathcal{O}\left(1 / \Lambda_{\chi}\right)$ corrections. These terms generate divergent chiral loops which contribute to the MMs. Their renormalization requires the introduction of higher-order operators. In the case of $S$ we find that all divergences and scale dependence to $\mathcal{O}\left(1 / \Lambda_{\chi}^{2}\right)$ can be absorbed in a redefinition of only one $\mathcal{O}\left(1 / \Lambda_{\chi}\right)$ coupling. Our computations and results are presented in Sec. III. The magnetic moments of the $T$ baryons are analyzed in Ref. [15]. However, this analysis 
does not include all meson loops and the needed counterterms are not taken into account. In Sec. IV we provide a consistent calculation of the $T$ magnetic moments to order $\mathcal{O}\left(1 /\left(m_{Q} \Lambda_{\chi}^{2}\right)\right)$. Finally, Sec. V summarizes our conclusions.

\section{HHCPT FORMALISM FOR MAGNETIC MOMENTS}

The light degrees of freedom in the ground state of a baryon with one heavy quark can be either in a $s_{l}=0$ or in a $s_{l}=1$ configuration. The first one corresponds to $J^{P}=\frac{1}{2}^{+}$ baryons, which are annihilated by $T_{i}(v)$ fields which transform as a $\overline{\mathbf{3}}$ under the chiral $\mathrm{SU}(3)_{L+R}$ and as a doublet under the HQET SU(2) $)_{v}$. In the second case, $s_{l}=1$, the spin of the heavy quark and the light degrees of freedom combine together to form $J^{P}=3 / 2^{+}$and $J=1 / 2^{+}$baryons which are degenerate in mass in the $m_{Q} \rightarrow \infty$ limit. The spin- $\frac{3}{2}$ ones are annihilated by the Rarita-Schwinger field $S_{\mu}^{* i j}(v)$, while the spin- $\frac{1}{2}$ baryons are destroyed by the Dirac field $S^{i j}(v)$. It is very useful to combine both operators into the so-called superfield [8]

$$
\begin{aligned}
& S_{\mu}^{i j}(v)=\sqrt{\frac{1}{3}}\left(\gamma_{\mu}+v_{\mu}\right) \gamma^{5} S^{i j}(v)+S_{\mu}^{* i j}(v), \\
& \bar{S}_{i j}^{\mu}(v)=-\sqrt{\frac{1}{3}} \bar{S}_{i j}(v) \gamma^{5}\left(\gamma^{\mu}+v^{\mu}\right)+\bar{S}_{i j}^{* \mu}(v),
\end{aligned}
$$

which transforms as a 6 under $\mathrm{SU}(3)_{L+R}$ and as a doublet under $\mathrm{SU}(2)_{v}$ and is symmetric in the $i, j$ indices.

The particle assignement for the $J=1 / 2$ charmed baryons of the $\overline{\mathbf{3}}$ and $\mathbf{6}$ representations is

$\left(T_{1}, T_{2}, T_{3}\right)=\left(\Xi_{c}^{0},-\Xi_{c}^{+}, \Lambda_{c}^{+}\right)$,

$$
S^{i j}=\left(\begin{array}{ccc}
\Sigma_{c}^{++} & \sqrt{\frac{1}{2}} \Sigma_{c}^{+} & \sqrt{\frac{1}{2}} \Xi_{c}^{+^{\prime}} \\
\sqrt{\frac{1}{2}} \Sigma_{c}^{+} & \Sigma_{c}^{0} & \sqrt{\frac{1}{2}} \Xi_{c}^{0^{\prime}} \\
\sqrt{\frac{1}{2}} \Xi_{c}^{+^{\prime}} & \sqrt{\frac{1}{2}} \Xi_{c}^{0^{\prime}} & \Omega_{c}^{0}
\end{array}\right),
$$

and the corresponding $b$ baryons are

$$
\begin{aligned}
&\left(T_{1}, T_{2}, T_{3}\right)=\left(\Xi_{b}^{-},-\Xi_{b}^{0}, \Lambda_{b}^{0}\right), \\
& S^{i j}=\left(\begin{array}{ccc}
\Sigma_{b}^{+} & \sqrt{\frac{1}{2}} \Sigma_{b}^{0} & \sqrt{\frac{1}{2}} \Xi_{b}^{0^{\prime}} \\
\sqrt{\frac{1}{2}} \Sigma_{b}^{0} & \Sigma_{b}^{-} & \sqrt{\frac{1}{2}} \Xi_{b}^{-^{\prime}} \\
\sqrt{\frac{1}{2}} \Xi_{b}^{0^{\prime}} & \sqrt{\frac{1}{2}} \Xi_{b}^{-^{\prime}} & \Omega_{b}^{-}
\end{array}\right) .
\end{aligned}
$$

The $J=3 / 2$ partners of the baryons of Eqs. (5) and (7) have the same $\mathrm{SU}(3)_{V}$ assignment in $S_{\mu}^{* i j}$.

The lowest-order chiral Lagrangian describing the soft hadronic and electromagnetic interactions of these baryons in the infinite heavy quark mass limit is given by [7]

$$
\begin{aligned}
\mathcal{L}^{(0)}= & -i \bar{S}_{i j}^{\mu}(v \cdot D) S_{\mu}^{i j}+\Delta_{S T} \bar{S}_{i j}^{\mu} S_{\mu}^{i j}+i \bar{T}^{i}(v \cdot D) T_{i} \\
& +i g_{2} \varepsilon_{\mu \nu \sigma \lambda} \bar{S}_{i k}^{\mu} v^{\nu}\left(\xi^{\sigma}\right)_{j}^{i}\left(S^{\lambda}\right)^{j k}+g_{3}\left[\epsilon_{i j k} \bar{T}^{i}\left(\xi^{\mu}\right)_{l}^{j} S_{\mu}^{k l}\right. \\
& \left.+\epsilon^{i j k} \bar{S}_{k l}^{\mu}\left(\xi_{\mu}\right)_{j}^{l} T_{i}\right] .
\end{aligned}
$$

In this formula, the heavy baryon covariant derivatives are

$$
\begin{aligned}
D^{\mu} S_{\nu}^{i j}= & \partial^{\mu} S_{\nu}^{i j}+\left(\Gamma^{\mu}\right)_{k}^{i} S_{\nu}^{k j}+\left(\Gamma^{\mu}\right)_{k}^{j} S_{\nu}^{i k}-i e \mathcal{A}^{\mu}\left[Q_{Q} S_{\nu}^{i j}+Q_{k}^{i} S_{\nu}^{k j}\right. \\
& \left.+Q_{k}^{j} S_{\nu}^{i k}\right] \\
D^{\mu} T_{i}= & \partial^{\mu} T_{i}-T_{j}\left(\Gamma^{\mu}\right)_{i}^{j}-i e \mathcal{A}^{\mu}\left[Q_{Q} T_{i}-T_{j} Q_{i}^{j}\right]
\end{aligned}
$$

where $\mathcal{A}^{\mu}$ is the electromagnetic current, $Q_{Q}$ is the heavy quark charge, the light quark charge matrix $Q$ is

$$
\mathbf{Q}=\left(\begin{array}{lll}
\frac{2}{3} & & \\
& -\frac{1}{3} & \\
& & -\frac{1}{3}
\end{array}\right),
$$

and the Goldstone fields appear through axial-vector, $\xi_{\mu}$, and vector, $\Gamma_{\mu}$, currents

$$
\begin{gathered}
\xi_{\mu}=i\left(\xi D_{\mu} \xi^{\dagger}-\xi^{\dagger} D_{\mu} \xi\right) / 2, \\
\Gamma_{\mu}=\left(\xi D_{\mu} \xi^{\dagger}+\xi^{\dagger} D_{\mu} \xi\right) / 2
\end{gathered}
$$

with

$$
D^{\mu} \xi=\partial^{\mu} \xi-i e \mathcal{A}^{\mu}[Q, \xi]
$$

Because of the different spin configuration of the light degrees of freedom, there is an intrinsic mass difference, $\Delta_{S T}$ $\equiv M_{S}-M_{T}$, among the $S^{(*)}$ and the $T$ baryons.

Notice that a direct coupling of the pseudo Goldstone bosons to the $\overline{\mathbf{3}}$ baryons is forbidden at the lowest order in $1 / \Lambda_{\chi}$.

As can be seen, there are no MM terms in the lowestorder Lagrangian in Eq. (8). Therefore, the contributions to the MMs come from the following.

(1) The next order in the baryon chiral Lagrangian [7]

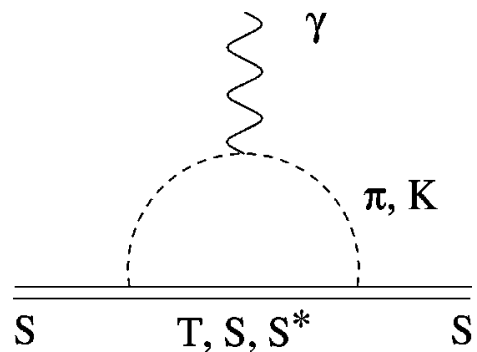

FIG. 1. Meson loops contributing to $S$-baryon MMs. 
TABLE I. Contributions to magnetic moments of spin- $\frac{1}{2} c$ and $b$ baryons $\left(s_{t}=1\right)$.

\begin{tabular}{ccccc}
\hline \hline$c$ quark & $b$ quark & $\mu_{x}$ & $\mu_{g_{3}}$ & $\mu_{g_{2}}$ \\
\hline$\Sigma_{c}^{++}$ & $\Sigma_{b}^{+}$ & 2 & $1+m_{\pi} / m_{K}$ & $I_{\pi}+I_{K}$ \\
$\Sigma_{c}^{+}$ & $\Sigma_{b}^{0}$ & $1 / 2$ & $1 / 2$ & $I_{K} / 2$ \\
$\Sigma_{c}^{0}$ & $\Sigma_{b}^{-}$ & -1 & $-m_{\pi} / m_{K}$ & $-I_{\pi}$ \\
$\Xi_{c}^{0^{\prime}}$ & $\Xi_{b}^{-{ }^{\prime}}$ & -1 & $-\left(1+m_{\pi} / m_{K}\right) / 2$ & $-\left(I_{\pi}+I_{K}\right) / 2$ \\
$\Xi_{c}^{+^{\prime}}$ & $\Xi_{b}^{0^{\prime}}$ & $1 / 2$ & $m_{\pi} /\left(2 m_{K}\right)$ & $I_{\pi} / 2$ \\
$\Omega_{c}^{0}$ & $\Omega_{b}^{-}$ & -1 & -1 & $-I_{K}$ \\
\hline \hline
\end{tabular}

$$
\begin{aligned}
\mathcal{L}^{(\text {long })}= & \frac{e}{\Lambda_{\chi}}\left\{i c_{S} \operatorname{tr}\left[\bar{S}_{\mu} Q S_{\nu}+\bar{S}_{\mu} S_{\nu} Q\right] F^{\mu \nu}\right. \\
& \left.+c_{S T}\left[\epsilon_{i j k} \bar{T}^{i} v_{\mu} Q_{l}^{j} S_{\nu}^{k l}+\epsilon_{i j k} \bar{S}_{\nu, k l} v_{\mu} Q_{j}^{l} T_{i}\right] \widetilde{F}^{\mu \nu}\right\},
\end{aligned}
$$

where the constants $c_{i}$ are all unknown. We will take $\Lambda_{\chi}$ $=4 \pi f_{\pi} \simeq 1.2 \mathrm{GeV}$, which fixes the normalization of the unknown couplings $c_{i}$.

(2) Terms of order $1 / m_{Q}$ from the heavy quark expansion which break both spin and flavor symmetries [7]:

$$
\begin{aligned}
\mathcal{L}^{(\text {short })}= & -\frac{1}{2 m_{Q}} \bar{S}_{i j}^{\lambda}(i D)^{2} S_{\lambda}^{i j}-\frac{e Q_{Q}}{4 m_{Q}} \bar{S}_{i j}^{\lambda} \sigma_{\mu \nu} S_{\lambda}^{i j} F^{\mu \nu} \\
& +\frac{1}{2 m_{Q}} \bar{T}^{i}(i D)^{2} T_{i}+\frac{e Q_{Q}}{4 m_{Q}} \bar{T}^{i} \sigma_{\mu \nu} T_{i} F^{\mu \nu} .
\end{aligned}
$$

(3) Chiral loops of Goldstone bosons coupled to photons, as described by the lowest-order Lagrangian.

\section{RESULTS FOR $S$ BARYONS $\left(s_{l}=1\right)$}

We define the magnetic moment operator for a spin- $\frac{1}{2}$ baryon $B$ and a spin- $\frac{3}{2}$ baryon $B_{\nu}^{*}$, respectively, as

$$
\begin{gathered}
-i e \mu(B) F^{\alpha \beta} \bar{B} \sigma_{\alpha \beta} B, \\
-i e \mu\left(B^{*}\right) F^{\alpha \beta} \bar{B}_{\mu}^{*} \sigma_{\alpha \beta} B^{* \mu} .
\end{gathered}
$$

The leading contributions from the light- and heavy-quark magnetic interactions are of order $\mathcal{O}\left(1 / \Lambda_{\chi}\right)$ and $\mathcal{O}\left(1 / m_{Q}\right)$, respectively. We compute the next-to-leading order (NLO) chiral corrections of order $\mathcal{O}\left(1 / \Lambda_{\chi}^{2}\right)$ which originate from the loop diagrams shown in Fig. 1.

The resulting MMs can be decomposed as

$$
\begin{aligned}
\mu\left(B^{(*)}\right)= & \frac{1}{72}\left(6 \frac{Q_{Q}}{m_{Q}} \mu_{H Q E}\left(B^{(*)}\right)+\frac{16 c_{s}}{\Lambda_{\chi}} \mu_{\chi}\left(B^{(*)}\right)\right. \\
& +3 g_{2}^{2} \frac{\Delta_{S T}}{\left(4 \pi f_{\pi}\right)^{2}} \mu_{g_{2}}\left(B^{(*)}\right) \\
& \left.-3 g_{3}^{2} \frac{m_{K}}{4 \pi f_{\pi}^{2}} \mu_{g_{3}}\left(B^{(*)}\right)\right)
\end{aligned}
$$

where $\mu_{i}(B)$ and $\mu_{i}\left(B^{*}\right)$ are related by

$$
\begin{aligned}
\frac{1}{3} \mu_{H Q E}\left(B^{*}\right) & =\mu_{H Q E}(B)=1, \\
\mu_{i}\left(B^{*}\right) & =-\frac{3}{2} \mu_{i}(B), \quad i=\chi, g_{2}, g_{3} .
\end{aligned}
$$

The values of the $\mu_{i}(B)$ contributions are reported in Table I for baryons containing a $Q$ quark $(Q=c, b)$ where

$$
\begin{aligned}
I_{i} \equiv & I\left(\Delta_{S T}, m_{i}\right)=2\left(-2+\log \frac{m_{i}^{2}}{\mu^{2}}\right) \\
& +2 \frac{\sqrt{\Delta_{S T}^{2}-m_{i}^{2}}}{\Delta_{S T}} \log \left(\frac{\Delta_{S T}+\sqrt{\Delta_{S T}^{2}-m_{i}^{2}}}{\Delta_{S T}-\sqrt{\Delta_{S T}^{2}-m_{i}^{2}}}\right) .
\end{aligned}
$$

We want to stress that due to flavor symmetry, the constants $c_{s}, g_{2}$, and $g_{3}$, and hence the values of $\mu_{\chi}, \mu_{g_{2}}$, and $\mu_{g_{3}}$, are the same for $c$ and $b$ baryons. The only difference is the contribution proportional to $\mu_{H Q E}$ due to the different electric charge of the $c, Q_{c}=+2 / 3$, and $b, Q_{b}=-1 / 3$, quarks [see Eq. (15)].

In Eq. (15) we have not considered contributions of order $\mathcal{O}\left(1 /\left(m_{Q} \Lambda_{\chi}\right)\right)$. For the $b$ baryons, these corrections can be safely neglected. For the $c$ baryons, however, a simple estimate shows that their contribution cannot be larger than, say, $15 \%$. In addition, the self-energy and loop diagrams with an insertion of the operator in Eq. (12) yield contributions of order $\mathcal{O}\left(1 / \Lambda_{\chi}^{3}\right)$ which again can be neglected because they are NNLO chiral corrections.

The results proportional to $g_{2}^{2}$ are obtained performing a one-loop integral (Fig. 1 with an $S$ baryon running in the loop) that has to be renormalized. The divergent part of the integral does not depend on the pion or kaon mass and is instead proportional to the mass of the baryon running in the loop. If one considers both pion and kaon loops, the divergent part respects the SU(3) structure of the chiral multiplet and can be canceled with an operator of the form

TABLE II. Theoretical estimates of $g_{2}$ and $g_{3}$.

\begin{tabular}{ccc}
\hline \hline Model & $g_{2}$ & $g_{3}$ \\
\hline Large $N_{c}[10]$ & 1.88 & 1.53 \\
Quark model [3] & 1.5 & 1.06 \\
Short distance QCD sum rule [12] & $0.83 \pm 0.23$ & $0.67 \pm 0.18$ \\
Light-cone QCD sum rules [13] & $1.56 \pm 0.3 \pm 0.3$ & $0.94 \pm 0.06 \pm 0.2$ \\
\hline \hline
\end{tabular}




$$
\frac{e}{\Lambda_{\chi}^{2}} \operatorname{tr}\left[\bar{S}_{\mu}\left(v \cdot D S_{\nu}\right) Q-\left(v \cdot D \bar{S}_{\mu}\right) S_{\nu} Q\right] F^{\mu \nu} .
$$

This is the most general dimension- 6 chiral and Lorentz invariant operator constructed out of $S_{\mu}^{i j}$ and $Q F_{\mu \nu}$, preserving parity and time-reversal invariance which contributes to MMs. When the equation of motion $\left[(v \cdot D) S_{\mu}=\Delta_{S T} S_{\mu}\right]$ is applied, its contribution is of the same form as the term proportional to $c_{s}$ in Eq. (12). Thus the local contribution from the operator in Eq. (18) can be taken into account, together with the lowest-order term in Eq. (12), through an effective coupling $c_{S}(\mu)$. The scale $\mu$ dependence of the loop integrals is exactly canceled by the corresponding dependence of the coefficient $c_{S}(\mu)$.

The contribution proportional to $g_{3}^{2}$ involves a loop integral in which a baryon of the $T$ multiplet is running in the loop. However, as we are in the limit of $m_{T} \rightarrow \infty$, no mass term for these $T$ baryons is present in the Lagrangian of Eq. (8). This means that the only massive particles running in the loop are the light mesons and the result of the integral is convergent and proportional to their mass.

Using Table I, one can derive the following linearly independent relations for the magnetic moments of spin- $\frac{1}{2}$ baryons containing a $c$ quark:

$$
\begin{aligned}
& \mu\left(\Sigma_{c}^{++}\right)+\mu\left(\Sigma_{c}^{0}\right)=2 \mu\left(\Sigma_{c}^{+}\right), \\
& \mu\left(\Sigma_{c}^{++}\right)+\mu\left(\Omega_{c}^{0}\right)=2 \mu\left(\Xi_{c}^{+^{\prime}}\right), \\
& \mu\left(\Sigma_{c}^{++}\right)+2 \mu\left(\Xi_{c}^{0^{\prime}}\right)=\mu\left(\Sigma_{c}^{0}\right)+2 \mu\left(\Xi_{c}^{+\prime}\right), \\
& \mu\left(\Sigma_{c}^{0}\right)+2 \mu\left(\Xi_{c}^{+\prime}\right)=\frac{1}{6 m_{c}} .
\end{aligned}
$$

Including the spin- $-\frac{3}{2}$ baryons, one can derive six more independent relations

$$
\begin{aligned}
\mu\left(\Sigma_{c}^{++*}\right)+\mu\left(\Sigma_{c}^{0 *}\right) & =2 \mu\left(\Sigma_{c}^{+*}\right), \\
\mu\left(\Sigma_{c}^{++*}\right)+\mu\left(\Omega_{c}^{0 *}\right) & =2 \mu\left(\Xi_{c}^{+^{\prime} *}\right), \\
\mu\left(\Sigma_{c}^{++*}\right)+2 \mu\left(\Xi_{c}^{0^{\prime} *}\right) & =\mu\left(\Sigma_{c}^{0 *}\right)+2 \mu\left(\Xi_{c}^{+{ }^{\prime} *}\right), \\
\mu\left(\Sigma_{c}^{0 *}\right)+2 \mu\left(\Xi_{c}^{0^{\prime} *}\right) & =3\left[\mu\left(\Sigma_{c}^{0}\right)+2 \mu\left(\Xi_{c}^{{ }^{\prime}}\right)\right], \\
\frac{2}{3} \mu\left(\Sigma_{c}^{++*}\right) & =\mu\left(\Sigma_{c}^{0}\right)+2 \mu\left(\Xi_{c}^{+\prime}\right)-\mu\left(\Sigma_{c}^{++}\right) \\
6 \mu\left(\Sigma_{c}^{+}\right)-4 \mu\left(\Sigma_{c}^{++}\right) & =-4 \mu\left(\Sigma_{c}^{+*}\right)+\frac{8}{3} \mu\left(\Sigma_{c}^{++*}\right) .
\end{aligned}
$$

The last three equations connect observables corresponding to spin- $-\frac{1}{2}$ and spin $-\frac{3}{2}$ baryons.

Moreover, it is easy to deduce ten analogous equations that relate baryons having a $b$ quark,

$$
\mu\left(\Sigma_{b}^{+}\right)+\mu\left(\Sigma_{b}^{-}\right)=2 \mu\left(\Sigma_{b}^{0}\right),
$$

$$
\begin{aligned}
\mu\left(\Sigma_{b}^{+}\right)+\mu\left(\Omega_{b}^{-}\right) & =2 \mu\left(\Xi_{b}^{0^{\prime}}\right), \\
\mu\left(\Sigma_{b}^{+}\right)+2 \mu\left(\Xi_{b}^{-{ }^{\prime}}\right) & =\mu\left(\Sigma_{b}^{-}\right)+2 \mu\left(\Xi_{b}^{0^{\prime}}\right), \\
\mu\left(\Sigma_{b}^{-}\right)+2 \mu\left(\Xi_{b}^{0^{\prime}}\right) & =-\frac{1}{12 m_{b}}, \\
\mu\left(\Sigma_{b}^{+*}\right)+\mu\left(\Sigma_{b}^{-*}\right) & =2 \mu\left(\Sigma_{b}^{0 *}\right), \\
\mu\left(\Sigma_{b}^{+*}\right)+\mu\left(\Omega_{b}^{-*}\right) & =2 \mu\left(\Xi_{b}^{0^{\prime} *}\right), \\
\mu\left(\Sigma_{b}^{+*}\right)+2 \mu\left(\Xi_{b}^{-{ }^{\prime}} *\right) & =\mu\left(\Sigma_{b}^{-*}\right)+2 \mu\left(\Xi_{b}^{0^{\prime} *}\right), \\
\mu\left(\Sigma_{b}^{-*}\right)+2 \mu\left(\Xi_{b}^{-\prime} *\right) & =3\left[\mu\left(\Sigma_{b}^{-}\right)+2 \mu\left(\Xi_{b}^{0^{\prime}}\right)\right], \\
\frac{2}{3} \mu\left(\Sigma_{b}^{+*}\right) & =\mu\left(\Sigma_{b}^{-}\right)+2 \mu\left(\Xi_{b}^{0^{\prime}}\right)-\mu\left(\Sigma_{b}^{+}\right), \\
6 \mu\left(\Sigma_{b}^{0}\right)-4 \mu\left(\Sigma_{b}^{+}\right) & =-4 \mu\left(\Sigma_{b}^{0 *}\right)+\frac{8}{3} \mu\left(\Sigma_{b}^{+*}\right),
\end{aligned}
$$

and two independent equations that relate $b$ and $c$ baryons,

$$
\begin{gathered}
\mu\left(\Sigma_{b}^{0}\right)-\mu\left(\Sigma_{b}^{+}\right)=\mu\left(\Sigma_{c}^{+}\right)-\mu\left(\Sigma_{c}^{++}\right), \\
\mu\left(\Sigma_{c}^{++}\right)-\frac{1}{3} \mu\left(\Sigma_{c}^{++*}\right)=\mu\left(\Sigma_{b}^{+}\right)-\frac{1}{3} \mu\left(\Sigma_{b}^{+*}\right) .
\end{gathered}
$$

From Table I, we see that the order $\mathcal{O}\left(1 / \Lambda_{\chi}\right)$ and $\mathcal{O}\left(1 / \Lambda_{\chi}^{2}\right)$ contributions cancel in the sum of all baryon MMs, within the sextet. Therefore, the average over the baryon moments measures the MMs of the heavy quark,

$$
\left\langle\mu\left(S_{Q}\right)\right\rangle=\frac{1}{3}\left\langle\mu\left(S_{Q}^{*}\right)\right\rangle=\frac{Q_{Q}}{12 m_{Q}} .
$$

Notice also that we can construct other combinations such that $c_{s}, g_{2}^{2}$, and $g_{3}^{2}$ contributions cancel:

$$
\begin{aligned}
\mu\left(\Sigma_{c}^{++}\right)+\mu\left(\Sigma_{c}^{0}\right)+\mu\left(\Omega_{c}^{0}\right) & =\mu\left(\Sigma_{c}^{+}\right)+\mu\left(\Xi_{c}^{0^{\prime}}\right)+\mu\left(\Xi_{c}^{+^{\prime}}\right) \\
& =\frac{1}{6} \frac{\mu_{H Q E}\left(B^{(*)}\right)}{m_{c}}, \\
\mu\left(\Sigma_{b}^{+}\right)+\mu\left(\Sigma_{b}^{-}\right)+\mu\left(\Omega_{b}^{-}\right) & =\mu\left(\Sigma_{b}^{0}\right)+\mu\left(\Xi_{b}^{-\prime}\right)+\mu\left(\Xi_{b}^{0^{\prime}}\right) \\
& =-\frac{1}{12} \frac{\mu_{H Q E}\left(B^{(*)}\right)}{m_{b}} .
\end{aligned}
$$

If one has a numerical estimate of the couplings $g_{2}$ and $g_{3}$, it is possible to derive a scale-independent relation between any couple of baryons. The combination

$$
\mu\left(B_{1}\right)-\frac{\mu_{\chi}\left(B_{1}\right)}{\mu_{\chi}\left(B_{2}\right)} \mu\left(B_{2}\right)
$$




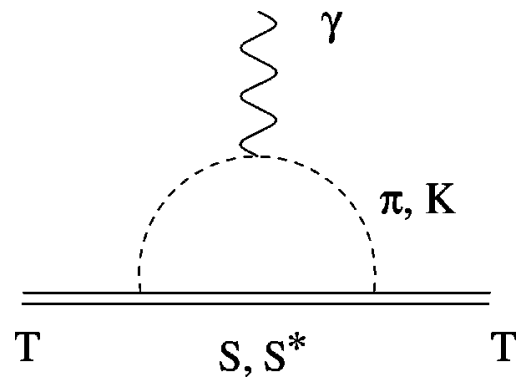

FIG. 2. Meson loops contributing to T-baryon MMs.

is independent of the unknown coupling $c_{S}(\mu)$ and can then be predicted. For instance,

$$
\begin{aligned}
\mu\left(\Sigma_{b}^{+}\right)+2 \mu\left(\Sigma_{b}^{-}\right)= & \frac{1}{24} \frac{g_{3}^{2}}{4 \pi f_{\pi}^{2}}\left(m_{K}-m_{\pi}\right) \\
& -\frac{\Delta_{S T}}{24} \frac{g_{2}^{2}}{\left(4 \pi f_{\pi}\right)^{2}}\left(I_{K}-I_{\pi}\right)-\frac{1}{12 m_{b}} .
\end{aligned}
$$

The couplings $g_{2}$ and $g_{3}$ have been calculated theoretically. In Table II we report the results of these computations.

There exists an experimental measurement of $g_{3}$ from CLEO coming from the decay $\Sigma_{c}^{*} \rightarrow \Lambda_{c} \pi[9,10], g_{3}$ $=\sqrt{3}(0.57 \pm 0.10)$. The direct measurement of $g_{2}$ is not possible at present. However, the quark model relates its value to $g_{3}[11]$, yielding $g_{2}=1.40 \pm 0.25$.

In order to get a numerical estimate of the left-hand side of Eq. (26), we set $g_{2}=1.5 \pm 0.3$ and $g_{3}=0.99 \pm 0.17$ and the rest of the constants as in Table III. We find, for our best estimate of Eq. (26),

$$
\mu\left(\Sigma_{b}^{+}\right)+2 \mu\left(\Sigma_{b}^{-}\right)=0.23 \pm 0.09 \mathrm{GeV}^{-1}
$$

\section{RESULTS FOR $T$ BARYONS $\left(s_{l}=0\right)$}

As the light quarks of the $T$ baryons are in a $s_{l}=0$ configuration, the contributions to the magnetic moments of these hadrons are $1 / m_{Q}$ suppressed [4]. The leading term is of the form $\mu_{H Q E} / m_{Q}$, and the first chiral corrections are of order $\mathcal{O}\left(1 /\left(m_{Q} \Lambda_{\chi}\right)\right)$ and come from [14]

$$
\mathcal{L}_{\text {(long) }}^{\prime}=\frac{c_{T}}{4 m_{Q}} \frac{e}{\Lambda_{\chi}} \bar{T}^{i} \sigma_{\mu \nu} Q_{i j} T^{j} F^{\mu \nu} .
$$

The contributions of order $\mathcal{O}\left(1 /\left(m_{Q} \Lambda_{\chi}^{2}\right)\right)$ have a different origin.

(1) There is a divergent contribution [14] coming from Eq. (8) through the chiral loops shown in Fig. 2, which is proportional to the explicit mass splitting,

$$
\Delta M_{Q}=3 \frac{\lambda_{2 S}}{m_{Q}}
$$

for the spin- $\frac{1}{2}$ and spin- $\frac{3}{2}$ parts of $S$ baryons [15]

(2) In addition, one can consider a spin-symmetrybreaking operator of $\mathcal{O}\left(1 / m_{Q}\right)$,
TABLE III. Constants used in numerical estimates.

\begin{tabular}{cc}
\hline \hline$f_{\pi}$ & $93 \mathrm{MeV}$ \\
$m_{\pi}$ & $140 \mathrm{MeV}$ \\
$m_{K}$ & $496.7 \mathrm{MeV}$ \\
$\Delta_{S T}$ & $168 \mathrm{MeV}$ \\
$m_{c}$ & $1.3 \mathrm{GeV}$ \\
$m_{b}$ & $4.8 \mathrm{GeV}$ \\
\hline
\end{tabular}

$$
\mathcal{L}^{\prime}=\frac{g^{\prime}}{m_{Q}}\left[\epsilon_{i j k} \bar{T}^{i} \sigma^{\mu \nu}\left(\xi_{\mu}\right)_{l}^{j} S_{\nu}^{k l}+\epsilon^{i j k} \bar{S}_{k l}^{\mu} \sigma_{\mu \nu}\left(\xi^{\nu}\right)_{j}^{l} T_{i}\right],
$$

which gives rise to divergent loop diagrams, as the one in Fig. 2, where one of the vertices is proportional to $g^{\prime}$.

(3) Further, there are finite contributions of the same order coming from the SU(3)-breaking operators

$$
e \frac{\omega_{1}}{4 m_{Q} \Lambda_{\chi}^{2}} \bar{T}^{i} \sigma_{\mu \nu} Q_{i l} \chi_{j}^{l} T^{j} F^{\mu \nu}+e \frac{\omega_{2}}{4 m_{Q} \Lambda_{\chi}^{2}} Q_{Q} \bar{T}^{i} \sigma_{\mu \nu} \chi_{i j} T^{j} F^{\mu \nu}
$$

where, in the limit of exact isospin symmetry,

$$
\chi=\left(\begin{array}{ccc}
m_{\pi}^{2} & & \\
& m_{\pi}^{2} & \\
& & 2 m_{K}^{2}-m_{\pi}^{2}
\end{array}\right) .
$$

As in the case of the $S$ baryons, when all Goldstone boson loops are included, the scale $\mu$ dependence of the result of Fig. 2 is canceled by the corresponding dependence of an effective $c_{T}(\mu)$. Neither the interaction term of Eq. (30) nor the finite terms of Eq. (31) were taken into account in Ref. [14].

Similarly to what we have done in the previous paragraph, we write the magnetic moment of $T$ baryons as

$$
\begin{aligned}
\mu(B)= & \frac{1}{24 m_{Q}}\left(-6 Q_{Q} \mu_{H Q E}(B)-\frac{c_{T}}{\Lambda_{\chi}} \mu_{T}(B)\right. \\
& +g_{3}^{2} \frac{3 \lambda_{2 S}}{\left(4 \pi f_{\pi}\right)^{2}} \mu_{g_{3}}(B)+6 g_{3} g^{\prime} \frac{\Delta_{S T}}{\left(4 \pi f_{\pi}\right)^{2}} \mu_{g^{\prime}}(B) \\
& \left.+2 \frac{\omega_{1} m_{K}^{2}}{\Lambda_{\chi}^{2}} \mu_{\chi_{1}}(B)-6 Q_{Q} \frac{\omega_{2} m_{K}^{2}}{\Lambda_{\chi}^{2}} \mu_{\chi_{2}}(B)\right)
\end{aligned}
$$

The values of the $\mu_{i}$ are written in Table IV where

$$
J_{i}=\frac{\partial}{\partial \Delta_{S T}}\left[\Delta_{S T} I\left(\Delta_{S T}, m_{i}\right)\right]
$$

Corrections to our results for $T$ baryons are of order $\mathcal{O}\left(1 / m_{Q}^{2}\right)$ and hence negligible.

By eliminating the unknown coupling constants, one can deduce two independent relations among the magnetic moments of both $T$ multiplets: 
TABLE IV. Contributions to magnetic moments of spin- $\frac{1}{2} T$-baryons $\left(s_{l}=0\right)$.

\begin{tabular}{ccccccc}
\hline \hline$c$ quark & $b$ quark & $\mu_{T}$ & $\mu_{g_{3}}$ & $\mu_{g^{\prime}}$ & $\mu_{\chi_{1}}$ & $\mu_{\chi_{2}}$ \\
\hline$\Xi_{c}^{0}$ & $\Xi_{b}^{-}$ & 4 & $J_{\pi}+J_{K}$ & $I_{\pi}+I_{K}$ & $-2 m_{\pi}^{2} / m_{K}^{2}$ & $m_{\pi}^{2} / m_{K}^{2}$ \\
$\Xi_{c}^{+}$ & $\Xi_{b}^{0}$ & -2 & $-J_{\pi}$ & $-I_{\pi}$ & $m_{\pi}^{2} / m_{K}^{2}$ & $m_{\pi}^{2} / m_{K}^{2}$ \\
$\Lambda_{c}^{+}$ & $\Lambda_{b}^{0}$ & -2 & $-J_{K}$ & $-I_{K}$ & $2-m_{\pi}^{2} / m_{K}^{2}$ & $2-m_{\pi}^{2} / m_{K}^{2}$ \\
\hline \hline
\end{tabular}

$$
\begin{aligned}
m_{b} \mu\left(\Xi_{b}^{-}\right)-m_{c} \mu\left(\Xi_{c}^{0}\right)= & m_{b} \mu\left(\Xi_{b}^{0}\right)-m_{c} \mu\left(\Xi_{c}^{+}\right), \\
m_{b} \mu\left(\Lambda_{b}^{0}\right)-m_{c} \mu\left(\Lambda_{c}^{+}\right)-\frac{1}{4}= & \left(2 \frac{m_{K}^{2}}{m_{\pi}^{2}}-1\right)\left[m_{b} \mu\left(\Xi_{b}^{-}\right)\right. \\
& \left.-m_{c} \mu\left(\Xi_{c}^{0}\right)-\frac{1}{4}\right] .
\end{aligned}
$$

In the absence of the SU(3)-breaking operators in Eq. (31), the average baryon MM over the $T$ multiplet would be equal to the heavy quark MM [14]. The result is, however, corrected by contributions proportional to the unknown couplings $\omega_{1}$ and $\omega_{2}:{ }^{1}$

$$
\begin{aligned}
\left\langle\mu\left(T_{Q}\right)\right\rangle= & -\frac{1}{4 m_{c}}\left[Q_{Q}+\frac{2 \omega_{1} m_{K}^{2}}{9 \Lambda_{\chi}^{2}}\left(1-\frac{m_{\pi}^{2}}{m_{K}^{2}}\right)\right. \\
& \left.-Q_{Q} \frac{\omega_{2} m_{K}^{2}}{3 \Lambda_{\chi}^{2}}\left(2+\frac{m_{\pi}^{2}}{m_{K}^{2}}\right)\right] .
\end{aligned}
$$

\section{CONCLUSIONS}

The magnetic moments of triplet and sextet heavy baryons have been computed in the HHCPT. The calculation of

\footnotetext{
${ }^{1}$ Notice that our definition of MMs differs from the one in Ref.
} [14] by a factor of $-1 / 4$. the $S^{(*)}$-baryon MMs at order $\mathcal{O}\left(1 / \Lambda_{\chi}^{2}\right)$ involves only one new arbitrary constant $c_{S}$. Thus it is possible to derive relations among the MMs of the hadrons in the same sextet where all masses and effective couplings are eliminated. As a result of heavy quark symmetry, the MMs of the $S$ and $S^{*}$ sextets are also related. Moreover, as $c$ and $b$ baryons are described by the same arbitrary constants, we can connect the MMs of the two kinds of hadrons. The average over one sextet equals the corresponding heavy quark MM.

In the case of $T$ baryons, the first corrections appear at order $\mathcal{O}\left(1 /\left(m_{Q} \Lambda_{\chi}^{2}\right)\right)$ and four arbitrary constants are required. Then we are left with only two independent relations which combine $c$ and $b$ triplets and contain $m_{c}$ and $m_{b}$. The average over one triplet equals the heavy quark MM only in the absence of SU(3)-breaking operators.

The measure of the magnetic moments of heavy baryons represents an experimental challenge. Nevertheless, several groups are contemplating the possibility of performing it in the near future (BTeV, SELEX) [16].

\section{ACKNOWLEDGMENTS}

I. S. wants to thank A. Della Riccia Foundation (Florence, Italy) for support. M. C. B. is indebted to the Spanish Ministry of Education and Culture financial support. This work has been supported in part by the European Union TMR Network "EURODAPHNE" (Contract No. ERBFMXCT98-0169), by DGESIC, Spain (Grant No. PB97-1261) and by CICYT (Grant No. AEN-96/1718).
[1] M. Wise, Phys. Rev. D 45, R2188 (1992).

[2] G. Burdman and J. Donoghue, Phys. Lett. B 280, 287 (1992).

[3] T. M. Yan et al., Phys. Rev. D 46, 1148 (1992); 55, 5851(E) (1997).

[4] P. Cho, Phys. Lett. B 285, 145 (1992).

[5] S. Weinberg, Physica A 96, 327 (1979); J. Gasser and H. Leutwyler, Nucl. Phys. B250, 456 (1985); B250, 517 (1985); B250, 539 (1985); A. Pich, Rep. Prog. Phys. 58, 563 (1995) and references therein.

[6] For reviews see, for instance, H. Georgi, Report No. HUTP91-A039, Boulder TASI 91:0589-630; B. Grinstein, Annu. Rev. Nucl. Part. Sci. 42, 101 (1992); M. Neubert, Phys. Rep. 245, 259 (1994); in ' 'Heavy flavours II,'” edited by A. J. Buras and M. Lindner, p. 239; M. B. Wise, in Probing the Standard Model of Particle Interactions (Proc. Les Houches Summer School, 1997), edited by R. Gupta et al. (North-Holland, Amsterdam, 1999), Vol. II, p. 1051; A. Pich, in ibid., p. 999, and references therein.
[7] P. Cho and H. Georgi, Phys. Lett. B 296, 408 (1992); 300, 410(E) (1993).

[8] A. Falk, Nucl. Phys. B378, 79 (1992).

[9] CLEO Collaboration, G. Brandenburg et al. Phys. Rev. Lett. 78, 2304 (1997).

[10] Z. Guralnik, M. Luke, and A. V. Manohar, Nucl. Phys. B390, 474 (1993)

[11] H.-Y. Cheng, Phys. Lett. B 399, 281 (1997).

[12] A. Grozin and O. I. Yakovlev, Eur. Phys. J. C 2, 721 (1998).

[13] S.-L. Zhu and Y.-B. Dai, Phys. Lett. B 429, 72 (1998).

[14] M. J. Savage, Phys. Lett. B 326, 303 (1994).

[15] See, e.g., P. Cho, Nucl. Phys. B396, 183 (1993); B421, 683(E) (1994); E. Jenkins, Phys. Rev. D 54, 4515 (1996).

[16] L. Moroni and J. Russ (private communication); BTeV Collaboration, A. Kulyavtsev et al. in San Juan 1998, High Energy Physics, edited by J. F. Nieves (AIP, Woodbury, 1998), p. 479. 